\title{
DAC-Listen der Hilfeempfängerländer
}

\section{OpenEdition}

\section{Journals}

Electronic version

URL: http://journals.openedition.org/sjep/859

DOI: $10.4000 /$ sjep.859

ISSN: 1663-9677

\section{Publisher}

Institut de hautes études internationales et du développement

\section{Printed version}

Date of publication: 1 avril 2001

Number of pages: 461-464

ISSN: 1660-5926

\section{Electronic reference}

« DAC-Listen der Hilfeempfängerländer », Schweizerisches Jahrbuch für Entwicklungspolitik [Online], 20 | 2001, Online erschienen am: 02 September 2012, abgerufen am 08 September 2020. URL : http:// journals.openedition.org/sjep/859; DOI : https://doi.org/10.4000/sjep.859 


\title{
1. TEIL: ENTWICKLUNGSLÄNDER UND -GEBIETE (ÖFFENTLICHE ENTWICKLUNGSHILFE)
}

\section{Am wenigsten entwickelte Länder (LDC)}

Afghanistan
Angola
Äquatorialguinea
Äthiopien
Bangladesch
Benin
Bhutan
Burkina Faso
Burundi
Dschibuti
Eritrea
Gambia
Guinea
Guinea-Bissau
Haiti
Jemen

Kambodscha
Kap Verde
Kiribati
Komoren
Kongo, Demokratische Rep.
Laos
Lesotho
Liberia
Madagaskar
Malawi
Malediven
Mali
Mauretanien
Mosambik
Myanmar
Nepal

\author{
Niger \\ Ruanda \\ Salomon-Inseln \\ Sambia \\ São Tomé und Príncipe \\ Sierra Leone \\ Somalia \\ Sudan \\ Tansania \\ Togo \\ Tschad \\ Tuvalu \\ Uganda \\ Vanuatu \\ Westsamoa \\ Zentralafrikanische Rep.
}

Sonstige einkommensschwache Länder ESL (BSP pro Einwohner unter 765 Dollar 1995)

$\begin{array}{lll}{ }^{*} \text { Albanien } & \text { Guyana } & \text { Nicaragua } \\ { }^{*} \text { Armenien } & \text { Honduras } & \text { Nigeria } \\ { }^{*} \text { Aserbaidschan } & \text { Indien } & \text { Pakistan } \\ \text { Bosnien-Herzegowina } & \text { Kamerun } & \text { Senegal } \\ \text { China } & \text { Kenia } & \text { Simbabwe } \\ \text { Elfenbeinküste } & { }^{*} \text { Kirgistan } & \text { Sri Lanka } \\ { }^{*} \text { Georgien } & \text { Kongo, Demokratische Rep. } & { }^{*} \text { Tadschikistan } \\ \text { Ghana } & \text { Mongolei } & \text { Vietnam }\end{array}$

Länder mit mittlerem Einkommen (untere Einkommensstufe) LME/UES (BSP pro Einwohner 766-3035 Dollar 1995)

\begin{tabular}{|c|c|c|}
\hline Ägypten & Jamaika & Papua-Neuguinea \\
\hline Algerien & Jordanien & Paraguay \\
\hline Belize & Jugoslawien, Bundesrep. & Peru \\
\hline Bolivien & ${ }^{*}$ Kasachstan & Philippinen \\
\hline Botswana & Kolumbien & St. Vincent und Grenadinen \\
\hline Costa Rica & Korea, Demokratische Rep. & Surinam \\
\hline Dominikanische Republik & Kuba & Swasiland \\
\hline Dominica & Libanon & Syrien \\
\hline Ecuador & Marokko & Thailand \\
\hline El Salvador & Marshall-Inseln & +Timor \\
\hline Fidschi & Mazedonien & +Tokelau \\
\hline Gebiete unter & (ehemalige Jugoslawische Rep.) & Tonga \\
\hline palästinensischer Verwaltung & Mikronesien & Tunesien \\
\hline Grenada & ${ }^{*}$ Moldawien & Türkei \\
\hline Guatemala & Namibia & *Turkmenistan \\
\hline Indonesien & Niue & *Usbekistan \\
\hline Irak & Palagos-Inseln & Venezuela \\
\hline Iran & Panama & +Wallis und Futuna \\
\hline
\end{tabular}

* Mittel- und osteuropäische Länder (MOEL)/Gemeinschaft Unabhängiger Staaten (GUS)

+ Gebiete 


$\begin{array}{lll}\begin{array}{l}\text { Länder mit mittlerem Einkommen (obere Einkommensstufe) (BSP pro Einwohner 3036-9385 Dollar 1995) } \\ \text { +Anguilla }\end{array} & \text { Kroatien } & \text { +St. Helena } \\ \text { Antigua und Barbuda } & \text { Libyen' } & \text { St. Kitts und Nevis } \\ \text { Saudi-Arabien } & \text { Malaysia } & \text { St. Lucia } \\ \text { Argentinien } & \text { Malta } & \text { Seychellen } \\ \text { Bahrain } & \text { Mauritius } & \text { Slowenien } \\ \text { Barbados } & \text { +Mayotte } & \text { Südafrika } \\ \text { Brasilien } & \text { Mexiko } & \text { Trinidad und Tobago } \\ \text { Chile } & \text { +Montserrat } & + \text { Turks-und Caicos-Inseln } \\ \text { Cook-Inseln } & \text { Nauru } & \text { Uruguay } \\ \text { Gabun } & \text { Oman } & \end{array}$

Länder mit hohem Einkommen (BSP pro Einwohner über 9385 Dollar 1995)

+Niederl. Antillen'

+Gibraltar'

+Macao

+ Aruba'

+Französisch Polynesien'

+Jungferninseln' (brit.)

+Neukaledonien'

Korea, Demokratische Rep.'

Nördliche Marianen'

\section{TEIL: TRANSITIONSLÄNDER UND -GEBIETE (ÖFFENTLICHE HILFE)}

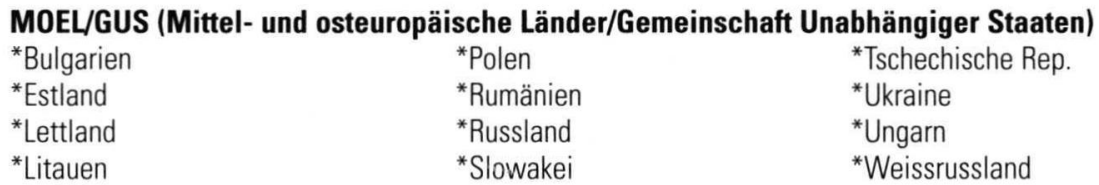

\section{Fortgeschrittenere Entwicklungsländer und -gebiete ${ }^{2}$}

+Bermuda-Inseln

Brunei

+Falkland-Inseln

Katar

+Cayman-Inseln

Hongkong, China

Kuweit

Singapur

Israel

Vereinigte Arabische Emirate Zypern

Anmerkung: Gemäss der vom DAC 1993 beschlossenen Politik setzt sich die DAC-Liste der Hilfeempfängerländer aus zwei Teilen zusammen. Die periodischen Überprüfungen nach festgesetzten Kriterien können dazu führen, dass bestimmte Empfängerländer von einem Teil in den anderen, besonders vom 1. in den 2. Teil verlegt werden (vgl. den Bericht "Coopération pour le développement $n, 1996$, p.A101).

Die Statistiken in diesem Bericht umfassen die letzten Daten für das Jahr 1996 und geben somit die DAC-Liste wieder. wie sie 1996 war. Die oben aufgeführte Liste tritt ab 1. Januar 1997 in Kraft. Die vorausgehenden Anmerkungen erklären unter anderem die Unterschiede zwischen den Listen für 1996 und für 1997.

Quelle: OCDE, Les dossiers du CAD, Coopération pour le développement, développement international, rapport 1999, 2000, vol. I, p. 289.

www.oecd.org/dac/htm/daclst97.htm.

1. All diese Länder und Gebiete sollen ab 1. Januar 2000 unter die fortgeschritteneren Länder eingestuft werden, sofern nicht eine Ausnahme beschlossen wird.

2. Die in Kursivschrift angegebenen Empfängerländer waren bis Ende $1996 \mathrm{im} \mathrm{1.} \mathrm{Teil} \mathrm{der} \mathrm{DAC-Liste} \mathrm{aufgeführt.} \mathrm{Bis} 1996$ ist die Hilfe für diese Länder in der öffentlichen Entwicklungshilfe für Länder mit hohem Einkommen enthalten. Sie wurden am 1. Januar 1997 in den 2. Teil umgestuft. Die anderen Empfängerländer in dieser Tabelle wurden ab 1. Januar 1996 in den 2. Teil umgestuft. Bis 1995 ist die Hilfe für diese Länder in der öffentlichen Entwicklungshilfe für Länder mit hohem Einkommen enthalten.

+ Gebiete.

* Mittel- und osteuropäische Länder (MOEL)/Gemeinschaft Unabhängiger Staaten (GUS). 


\section{TEIL: ENTWICKLUNGSLÄNDER UND -GEBIETE (ÖFFENTLICHE ENTWICKLUNGSHILFE)}

$\begin{array}{lll}\text { Am wenigsten entwickelte Länder (LDC) } & \\ \text { Afghanistan } & \text { Kambodscha } & \text { Niger } \\ \text { Angola } & \text { Kap Verde } & \text { Ruanda } \\ \text { Äquatorialguinea } & \text { Kiribati } & \text { Salomon-Inseln } \\ \text { Äthiopien } & \text { Komoren } & \text { Sambia } \\ \text { Bangladesch } & \text { Kongo, Demokratische Rep. } & \text { São Tomé und Príncipe } \\ \text { Benin } & \text { Laos } & \text { Sierra Leone } \\ \text { Bhutan } & \text { Lesotho } & \text { Somalia } \\ \text { Burkina Faso } & \text { Liberia } & \text { Sudan } \\ \text { Burundi } & \text { Madagaskar } & \text { Tansania } \\ \text { Dschibuti } & \text { Malawi } & \text { Togo } \\ \text { Eritrea } & \text { Malediven } & \text { Tschad } \\ \text { Gambia } & \text { Mali } & \text { Tuvalu } \\ \text { Guinea } & \text { Mauretanien } & \text { Uganda } \\ \text { Guinea-Bissau } & \text { Mosambik } & \text { Vanuatu } \\ \text { Haiti } & \text { Myanmar } & \text { Westsamoa } \\ \text { Jemen } & \text { Nepal } & \text { Zentralafrikanische Rep. }\end{array}$

Sonstige einkommensschwache Länder (BSP pro Einwohner unter 760 Dollar 1998)

${ }^{*}$ Armenien

*Aserbaidschan

China

Elfenbeinküste

Ghana

Honduras

Indien

Indonesien
Kamerun

Kenia

*Kirgistan

Kongo, Demokratische Rep.

Korea, Demokratische Rep.

*Moldawien

Mongolei

Nicaragua
Nigeria

+Osttimor

Pakistan

Senegal

Simbabwe

*Tadschikistan

*Turkmenistan

Vietnam

Länder mit mittlerem Einkommen (untere Einkommensstufe) (BSP pro Einwohner 761-3030 Dollar 1998)

$\begin{array}{lll}\text { Ägypten } & \text { Guyana } & \text { Papua-Neuguinea } \\ { }^{*} \text { Albanien } & \text { Irak } & \text { Paraguay } \\ \text { Algerien } & \text { Iran } & \text { Peru } \\ \text { Belize } & \text { Jamaika } & \text { Philippinen } \\ \text { Bolivien } & \text { Jordanien } & \text { Sri Lanka } \\ \text { Bosnien-Herzegowina } & \text { Jugoslawien, Bundesrep. } & \text { St. Vincent und Grenadinen } \\ \text { Costa Rica } & \text { *Kasachstan } & \text { Südafrika } \\ \text { Dominica } & \text { Kolumbien } & \text { Surinam } \\ \text { Dominikanische Republik } & \text { Kuba } & \text { Swasiland } \\ \text { Ecuador } & \text { Marokko } & \text { Syrien } \\ \text { El Salvador } & \text { Marshall-Inseln } & \text { Thailand } \\ \text { Fidschi } & \text { Mazedonien } & \text { +Tokelau } \\ \text { Gebiete unter } & \text { (ehemalige Jugoslawische Rep.) Tonga } \\ \text { palästinensischer Verwaltung } & \text { Mikronesien } & \text { Tunesien } \\ { }^{*} \text { Georgien } & \text { Namibia } & \text { *Usbekistan } \\ \text { Guatemala } & \text { Niue } & + \text { +Wallis und Futuna }\end{array}$

* Mittel- und osteuropäische Länder (MOEL)/Gemeinschaft Unabhängiger Staaten (GUS)

+ Gebiete 


$\begin{array}{lll}\text { Länder mit mittlerem Einkommen } & \text { (obere Einkommensstufe) (BSP pro Einwohner 3031-9360 Dollar 1998) } \\ \text { +Anguilla } & \text { Kroatien } & \text { Saudi-Arabien } \\ \text { Antigua und Barbuda } & \text { Libanon } & \text { Seychellen } \\ \text { Argentinien } & \text { Malaysia } & + \text { St. Helena } \\ \text { Bahrain } & \text { Mauritius } & \text { St Kitts und Nevis } \\ \text { Barbados } & + \text { Mayotte } & \text { St. Lucia } \\ \text { Botswana } & \text { Mexiko } & \text { Trinidad und Tobago } \\ \text { Brasilien } & \text { +Montserrat } & \text { Türkei } \\ \text { Chile } & \text { Nauru } & + \text { Turks-und Caicos-Inseln } \\ \text { Cook-Inseln } & \text { Oman } & \text { Uruguay } \\ \text { Gabun } & \text { Palau-Inseln } & \text { Venezuela } \\ \text { Grenada } & \text { Panama } & \end{array}$

Länder mit hohem Einkommen (BSP pro Einwohner über 9360 Dollar 1998)

Malta

Slowenien

\section{TEIL: TRANSITIONSLÄNDER UND -GEBIETE (ÖFFENTLICHE HILFE)}

\section{MOEL/GUS (Mittel- und osteuropäische Länder/Gemeinschaft Unabhängiger Staaten)}

*Bulgarien

${ }^{*}$ Estland

*Lettland

* Litauen
*Polen

*Rumänien

${ }^{*}$ Russland

${ }^{*}$ Slowakei
*Tschechische Rep.

*Ukraine

*Ungarn

*Weissrussland

\section{Fortgeschrittenere Entwicklungsländer und -gebiete}

+Aruba

Bahamas

+Bermuda-Inseln

Brunei

+Cayman-Inseln

Chinesisch Taipeh

+Falkland-Inseln

Französisch Polynesien
+Gibraltar

Hongkong, China

Israel

+Jungferninseln (brit.)

Katar

Korea, Rep.

Kuweit

Libyen
+Macao

+Neukaledonien

+Niederl. Antillen

Nördliche Marianen

Singapur

Vereinigte Arabische Emirate

Zypern

Anmerkung: Gemäss der vom DAC 1993 beschlossenen Politik setzt sich die DAC-Liste der Hilfeempfängerländer aus zwei Teilen zusammen. Die periodischen Überprüfungen nach festgesetzten Kriterien können dazu führen, dass bestimmte Empfängerländer von einem Teil in den anderen, besonders vom 1. in den 2. Teil verlegt werden (vgl. den Bericht "Coopération pour le développement", 1996, p.A101).

Quelle: OCDE, Les dossiers du CAD, Coopération pour le développement, développement international, rapport 1999, 2000, vol. I, p. 284.

www.oecd.org/dac/htm/daclst2000.htm.

1. Diese zwei Länder sollen ab 1. Januar 2003 unter die fortgeschritteneren Länder eingestuft werden, falls nicht eine Ausnahme beschlossen wird.

+ Gebiete.

* Mittel- und osteuropäische Länder (MOEL)/Gemeinschaft Unabhängiger Staaten (GUS). 